\title{
BMJ Open Institutional governance and responsiveness to antimicrobial resistance: a qualitative study of Australian hospital executives
}

\author{
Jennifer Broom (1) , ${ }^{1,2}$ Alex Broom, ${ }^{3}$ Katherine Kenny (D) , ${ }^{3}$ Jeffrey J. Post (D) , 4,5 \\ Pamela Konecny (iD) 6,7
}

To cite: Broom J, Broom A, Kenny K, et al. Institutional governance and responsiveness to antimicrobial resistance: a qualitative study of Australian hospital executives. BMJ Open 2021;11:e055215. doi:10.1136/ bmjopen-2021-055215

- Prepublication history for this paper is available online. To view these files, please visit the journal online (http://dx.doi. org/10.1136/bmjopen-2021055215).

Received 08 July 2021 Accepted 12 November 2021

D Check for updates

(c) Author(s) (or their employer(s)) 2021. Re-use permitted under CC BY-NC. No commercial re-use. See rights and permissions. Published by BMJ.

For numbered affiliations see end of article.

Correspondence to Dr Jennifer Broom; jennifer.broom@health.qld. gov.au

\section{ABSTRACT}

Objectives Despite escalating antimicrobial resistance (AMR), implementing effective antimicrobial optimisation within healthcare settings has been hampered by institutional impediments. This study sought to examine, from a hospital management and governance perspective, why healthcare providers may find it challenging to enact changes needed to address rising AMR.

Design Semistructured qualitative interviews around their experiences of antimicrobial stewardship (AMS) and responsiveness to the requirement for optimisation. Data were analysed using the framework approach.

Setting Two metropolitan tertiary-referral hospitals in Australia.

Participants Twenty hospital managers and executives from the organisational level of department head and above, spanning a range of professional backgrounds and in both clinical and non-clinical roles, and different professional streams were represented.

Results Thematic analysis demonstrated three key domains which managers and executives describe, and which might function to delimit institutional responsiveness to present and future AMR solutions. First, the primacy of 'political' priorities. AMR was perceived as a secondary priority, overshadowed by political priorities determined beyond the hospital by state health departments/ministries and election cycles. Second, the limits of accreditation as a mechanism for change. Hospital accreditation processes and regulatory structures were not sufficient to induce efficacious AMS. Third, a culture of acute problem 'solving' rather than future proofing. A culture of reactivity was described across government and healthcare institutions, precluding longer term objectives, like addressing the AMR crisis.

Conclusion There are dynamics between political and health service institutions, as well as enduring governance norms, that may significantly shape capacity to enact AMS and respond to AMR. Until these issues are addressed, and the field moves beyond individual behaviour modification models, antimicrobial misuse will likely continue, and stewardship is likely to have a limited impact.

\section{INTRODUCTION}

Antimicrobial resistance (AMR) is a current global crisis that will persist and accelerate
Strengths and limitations of this study

- Documents previously unrecognised influence of political priorities and governance structures which may significantly limit the power of antimicrobial stewardship activities.

- This study brings into focus levels of power and influence that have previously been opaque with a focus on the individual prescriber behaviour.

- Indicates the importance of integrating non-clinicians on processes and practices of implementation.

- Limited to the public health system in Australialikely to have cross-sector applicability but needs further examination in other settings.

without substantial change in our antimicrobial use. Antibiotic-resistant infections and the burden of mortality associated with them are increasing internationally. ${ }^{12}$ And yet internationally, effective action to preserve antimicrobials for the future has been peculiarly limited. Within Australian hospitals, antimicrobial use actually increased between 2016 and 2017 from 932.8 to 956.8 defined daily doses per 1000 occupied bed days and inappropriate prescribing is persistent over time at $21.4 \%$ of hospital prescriptions. ${ }^{3}{ }^{4}$ Internationally, antimicrobial stewardship (AMS) processes (aimed at the optimisation of antimicrobial use) have also had limited traction despite substantial resource allocation towards regulatory processes. ${ }^{5-7}$ As roll-out of AMS has, disappointingly, not resulted in significant changes in practice, even in highincome countries, international attention has begun to focus on the societal and human influences on antimicrobial use. ${ }^{8}$ The authors' and others' research have demonstrated the influence of broader social, cultural and systemic dimensions on antimicrobial use and stewardship. ${ }^{9-11}$ While the macro factors structuring individual prescribing decisions 
have now received significant attention, the health system governance dimensions have received little attention. Yet these systemic dimensions may be as, or more, significant than interactional influences. ${ }^{12}$

The AMS field internationally is still largely focused on finding the right 'AMS champions' - the right motivation (the right 'carrot' or 'stick') ${ }^{13}$ to empower clinicians to prescribe judiciously and push antimicrobial use in the right direction. ${ }^{1415}$ But the very systems within which attempts to enact change are occurring are highly complex and interwoven with economic and political priority setting. Such processes require critical examination to identify the way forward to effective stewardship and potent AMR solutions.

This study was designed to extend previous pilot work from the investigators, ${ }^{16}$ and examines, from the perspectives of a range of senior healthcare managers and executives from hospitals in Australia, the interplay of governance, managerial responsibility and clinical practice, with a view to understanding how institutional forms and priorities might shape antimicrobial optimisation practices. Previous studies have examined macrostructural drivers of AMR such as the global flows of pharmaceuticals and research and development, and microinteractional issues such as individual prescriber behaviour and interprofessional and intraprofessional issues have been examined by the authors and others. ${ }^{9}{ }^{17-21}$ This study examines the accounts of actors operating at the 'meso' level; the space where political policy and currency meet hospital governance and individual prescribers, and how complex dynamics in this level may critically determine or delimit action on AMR.

\section{Context: AMR/AMS within Australia}

The Australian government released its first AMR Strategy in 2015 and an implementation plan to support this strategy. This strategy included the regulatory requirements of the presence of AMS programmes within Australian hospitals. This study was performed in two metropolitan Australian Hospitals. AMS is a regulatory requirement for all Australian hospitals (public and private) and is assessed for hospital accreditation under the National Safety and Quality Health Service Standards. Hospitals are required to have an AMS policy, governance systems in place, and monitoring and quality improvement systems in place around antimicrobial prescribing. Stewardship programmes were present in both of the participating hospitals, including electronic decisionsupport systems for antibiotic approvals at both sites.

\section{METHODS}

This article draws on interviews with 20 hospital managers and executives from the organisational level of department head and above, which were conducted as part of a larger study into the social dynamics of AMR across two hospital sites in one major metropolitan area of Australia between 2018 and 2020. At each site, potential participants were purposively sampled and contacted via email with study information and an invitation to participate in a research interview. Overall, 50 hospital managers and executives were contacted and 20 interviews were completed before February 2020 when recruitment was suspended due to COVID-19. Of these, 12 participants were currently working in hybrid clinical-managerial roles, and 8 in exclusively executive roles. Interviews were conducted face-to-face, digitally audio-recorded and transcribed in full. Participants were all clinically trained and were currently employed in both clinical and nonclinical roles across a wide range of different professional streams. Interviews were semistructured and focused on the day-to-day practices of governance-in-action including questions about daily responsibilities, individual and institutional priority setting, accountability, determinations of value, how change is enacted, how performance is managed, the daily pressures of participants' roles and the short-term, mid-term and long-term objectives that drive hospital practice.

\section{Analysis}

The thematic analysis of the data was driven by the framework approach. This included full transcription and review of interviews, familiarisation with the data, coding, developing a working analytical framework, applying the analytical framework across the entire dataset and finally, developing an overall thematic interpretation of the data. ${ }^{22}$ Independent coding of the data was provided initially by members of the research team (author 1, author 2 and author 3), which was then cross-checked to facilitate the development of codes, categories and eventual themes. Analytic rigour was enhanced by searching for negative, atypical and conflicting or contradicting cases in coding and theme development. Inter-rater reliability was ensured by integrating research team members in the final analysis, including two infectious diseases specialists (author 4 and author 5 ).

\section{Patient and public involvement statement}

While this article comments on the perspectives of hospital executives and senior managers, this research also more broadly included patient and public involvement. Patients, carers, as well as stakeholders in legislative and policy and accreditation-related roles were also interviewed and/or observed along with a variety of healthcare workers both in and outside of the clinic.

\section{RESULTS}

\section{Participants}

Twenty managers and executives were interviewed (12 men and 8 women), from a range of positions from the organisational level of department head and above, across two metropolitan hospitals in Australia. Represented departments included surgery, oncology, haematology, respiratory medicine, neurology, intensive care, pharmacy and senior executive positions. 
Table 1 The political context of hospital governance

\begin{tabular}{ll}
\hline Indicative quotations \\
\hline Participants & Quote \\
\hline P 26 & ...you don't ever hear about the Ministry caring about patterns of [antimicrobial] prescribing. It's not something \\
that anyone above me ever appears to have cared about.
\end{tabular}

\section{Theme 1: the political context of hospital governance}

A significant theme in this study was the 'politics' that surround hospital governance. Executives perceived that they were held accountable for achieving targets that were prioritised by the Ministry of Health vis-à-vis those espoused by regulatory agencies or accreditation bodies. Given this, almost all the interviewees perceived AMS as a low priority for the government or the Ministry of Health. When reflecting on what was prioritised, the issue of public accountability (of the Ministry), and the health service accountability (to the Ministry) was perceived as paramount, leading to a focus on media sensitive performance indicators (such as emergency access targets), inpatient activity (which drove funding), and balances of services which improved outcomes and reduced costs. Interestingly, a number of participants did identify that appropriate antimicrobial use would improve hospital outcomes and potentially reduce costs associated with long-term-resistant organisms and adverse outcomes from infections; however, as one executive reflected, this loop was not perceived to be 'closed' in the minds of Government (and was a cost benefit 'too long term' for prioritisation in current government models-see theme three). Participants did reflect that it was a requirement to have a system in place for stewardship, but beyond that, at this point, there was little accountability for antimicrobial improvement outcomes (for indicative quotations on this theme see table 1 ).

\section{Theme 2: the limits of accreditation as a mechanism for change}

Accreditation standards for the hospital in terms of AMS were discussed. A minority of participants reflected on the increased attention on AMS via inclusion in the National Standards. However, the other participants reflected on the perceived limited power of accreditation to bring about meaningful change in prescribing, including that there was not a clear agreed requirement for AMS for accreditation, and that they had not seen 'strong language' from accreditors around AMS (which would be perceived as a powerful incentive for change). The focus on performance measures was perceived to direct attention away from issues of clinical leadership and influence which were perceived to have more power in changing practice. The lack of clarity around what was required for accreditation, in combination with variation between assessing accreditors in what attention was focused on, and a perceived lack of consequences for ineffective systems, led to a sense of inertia around instituting effective optimisation and thus responding to AMR (for indicative quotations on this theme see table 2 ).

\section{Theme 3: a culture of acute problem-solving rather than future-proofing}

The political flows of influence, and perceived limits to the power of accreditation processes, were accompanied by a third and vital macro characteristic - a ubiquitous and often implicit 'triage ideology' for maintaining institutional health. At all levels of management, participants reflected that the system was reactionary, and often in response to acute or media-sensitive events, and in a system with limited resources, this left little room for resource allocation for longer term issues such as addressing the risk of AMR. Although the majority of participants reflected on the increasing importance and threat of AMR, and expressed concern about the future, they discussed the lack of visibility of AMR and the importance of AMS in a system that was reactive to problems that were more acutely visible and more immediate. Some participants reflected on the high volume of clinical workload, which also limited the capacity of actors within the system to devote attention on longer term issues (for indicative quotations on this theme see table 3 ).

\section{DISCUSSION}

AMR is a clear and present danger. However, despite national strategies directed towards limiting AMR, significant change in use of antimicrobials in Australia and internationally is proving difficult to achieve. ${ }^{23} 24$ Increasingly, there is a need to examine the ideological, political and economic factors that limit antibiotic optimisation strategies despite substantial investment in such activities. There are relatively limited data on the role of politics and governance on antibiotic optimisation activities or the viability of AMR solutions 
Table 2 The limits of accreditation as a mechanism for change

\section{Indicative quotations}

\begin{tabular}{|c|c|}
\hline Participants & Quote \\
\hline P 23 & $\begin{array}{l}\text { Well, I don't think the accreditation system works necessarily very well. It all comes down to how good your } \\
\text { executive in the hospital is and what they're trying to achieve, what their vision is. }\end{array}$ \\
\hline P 22 & $\begin{array}{l}\text { The difficulty with AMS is what's the agreed requirement? It's not clear. We don't have a World Health } \\
\text { Organisation that said, "Okay, for surgical safety, you should do this checklist," and so an accreditor will just } \\
\text { come by and say, "Show us your whole bunch of charts and we'll check and see if the checklists have been } \\
\text { done properly," and then they're happy, or they talk to the staff and they say, "Tell me what you do to make } \\
\text { sure you've got the right patient and you're doing the right procedure on them." So, it's really cut and dry. }\end{array}$ \\
\hline P 20 & $\begin{array}{l}\text { So those Standards, those one to } 10 \text { are the key things that if we focused on those - well, we're focusing } \\
\text { on those, but we're focusing not quite on the right path. We need to focus more on what are the things } \\
\text { underneath that, systematically the behavioural things, like you talk about, is that we're afraid to escalate } \\
\text { because we don't want to be yelled at by Sir, so we don't when we're worried about a patient. It's the human } \\
\text { factor side of things of escalation. We fail to talk to one another because we're all too busy and we don't have } \\
\text { time. And we don't properly assess patients. We're rushing, we're busy, we're focused on KPIs. So, if we } \\
\text { focused on those three things, and following guidelines, we'd be a lot better off. }\end{array}$ \\
\hline
\end{tabular}

(for exceptions see ${ }^{1625}$ ). This study draws attention to broader systemic issues which are layered across the roll-out of stewardship within the Australian hospital environment, including the influence of government level priorities (which influence priority setting within hospitals) and regulatory processes.

The issues we have raised here align with the emphasis of recent work in implementation science and complexity science. ${ }^{26} 27$ That is, how microinterventions (ie, AMR based surveillance, restriction and improvement systems, often under the rubric of AMS, 'take hold'-or not, as the case may be-within an environment which is dynamic, complex and evolving). This includes the vagaries of politics and the implicit logics of institutional 'health' (vs what best serves individual health). Often, such spheres of implementation are opaque despite having a major effect on uptake of evidence based practice ${ }^{27}$ and in this case, AMR solutions. AMS in hospitals has to a large extent focused on the micro-such as individual adopter characteristics and/or intraorganisational characteristics such as low resource settings. ${ }^{28}$ This has meant little attention to the macrocontext such as political influence, endemic institutional logics of survival, viability of deployed legislative requirements (ie, once they 'trickle down' to institutions), and how all these factors manifest within organisations and their ability to respond to AMR.

This study begins to bring into focus reasons which may underlie the inertia of health systems in their AMR responses. In this study, participants describe responding to political priorities (characterised by risk averse, media sensitive, short-term outcomes) and regulated by a system of accreditation which is perceived to have limited traction in determining meaningful AMR progress within institutions, resulting in limited motivation for moving beyond tokenism in AMS responses. Internationally, the critical need for collaboration between government departments and hospitals to

Table 3 A culture of acute problem-solving rather than future-proofing

\section{Indicative quotations}

\section{Participants Quote}

P 20

It's all reaction. "Shit, the Minister is looking bad at the minute. It's on the front page of the paper. Quick, quick, quick. We've got to have a teleconference. We've got to pull everybody together." It's very reactive, and yet probably the issues have been around for a while. But as soon as it hits any media, it's all up in arms of, "Quick you're making the Minister look bad and where's the response?"

P 5 What our mandate is, is the here and now of acute medicine. The reality is if we could invest a little bit into the future, then we would reduce, for the future, the requirement for acute medicine right now. But how do you prioritise the resources that you've got that don't feel like they're enough to be able to deal with the here and now acute, to be able to allocate some to the future? And how do you find that balance? ...

Because antimicrobial resistance is one of those topics that is, I guess, a broader issue as opposed to specifically an individual for that person at that time. Yes, there's some studies, I think, that point to resistance even on a one-off one individual type scenario. But antibiotic resistance is one of those more global, bigger concepts, whereas sepsis, and the risk of dying from sepsis, is much more immediate, here and now, for both the individual, and perhaps even for the treating clinician. Because the person in front of you you're treating for potential sepsis, isn't going to exsanguinate from antibiotic microbial resistance at that time. Whereas they could do from sepsis. 
improve AMS has been identified. ${ }^{29}$ In addition, lack of coordinated government action on AMR has been documented. Examples include an extensive review of Canadian AMR policy identified a significant absence of policy approaches to antimicrobial regulation, and a lack of AMR engagement from professional associations and regulatory colleges. ${ }^{30}$ The limits of behavioural change-focused strategies such as educational interventions were highlighted in this report. The Italian picture echoes the same story; in a country with an extremely high burden of multiresistant bacteria and the largest number attributable deaths from resistant bacteria in Europe, a report by the European Centre for Disease Prevention and Control identified a lack of urgency about AMR and a tendency of stakeholders to avoid taking charge of the problem. Lack of leadership and institutional support, coordination and accountability for activities was also identified ${ }^{31}$ The UK's 5-year national action plan on AMR 2019-2024 does begin to define the broad changes required to address AMR and optimise antimicrobial use within the UK, and is beginning to examine system level strategies such as delinking funding of antibiotics from volume of antibiotics use. ${ }^{32}$ From our study, it would seem that the environment of AMS governance and prioritisation within Australia is not yet optimised to enable an effective response to AMR, and that a focus beyond prescriber behaviour, encompassing government priorities and clearly specified regulatory processes, would likely be necessary to effect change in hospital AMS processes. The imperative for clear governance is recognised at a government level in Australia, with the first priority of the recent Australian Antimicrobial Resistance Strategy being 'Clear Governance for Antimicrobial Resistance Initiatives'. ${ }^{33}$

This study was performed within two public metropolitan hospitals in Australia. It is therefore exploratory in nature and governance may differ across settings/ contexts. The qualitative nature elicits rich data on the experiences and perspectives of actors within the management of hospitals, but government and regulatory authority perspectives were not explored in this study and would be an extremely useful direction for future research.

\section{CONCLUSIONS}

There are complex political and regulatory dynamics which are interconnected with and influence antimicrobial use and optimisation within hospitals and which therefore impede AMS efforts to reduce inappropriate prescribing, a major driver of AMR. AMS activities when enacted in a system that prioritises reaction to crisis, risk avoidance, and financial performance indicators, may gain limited traction. Stewardship activities that focus on individual patient and prescriber behavioural change in antimicrobial prescribing, but do not address the higher influences and dynamics of system the actors are within, are likely to be significantly limited in power to generate longer term systemic changes in antimicrobial use.

\section{DISSEMINATION DECLARATION}

We plan to disseminate the results to hospital, community and patient organisations through internal reports and peer-review manuscript, as it becomes available.

\section{Author affiliations}

${ }^{1}$ Infectious Diseases Service, Sunshine Coast University Hospital, Sunshine Coast, Queensland, Australia

${ }^{2}$ School of Biomedical Sciences, The University of Queensland, Herston,

Queensland, Australia

${ }^{3}$ Sydney Centre for Healthy Societies, School of Social and Political Sciences, University of Sydney, Sydney, New South Wales, Australia

${ }^{4}$ Department of Infectious Diseases, Prince of Wales Hospital and Community Health Services, Randwick, New South Wales, Australia

${ }^{5}$ Prince of Wales Clinical School, University of New South Wales, Randwick, New South Wales, Australia

${ }^{6}$ Department of Infectious Diseases, Immunology \& Sexual Health, St George Hospital, Sydney, New South Wales, Australia

${ }^{7}$ St George and Sutherland Clinical School, University of New South Wales, Sydney, New South Wales, Australia

Twitter Jennifer Broom @jenniferkbroom, Alex Broom @BroomAlex and Katherine Kenny @kennykatherine

Acknowledgements The support of the Australasian Society for Infectious Diseases, the Australian Society for Antimicrobials and the New South Wales Clinical Excellence Commission in this work is appreciated.

Contributors Contributor $A B$ was principle investigator for the project, and carried out most of the project planning, design, governance, data collection and analysis, and writing of the manuscript. $A B$ was also the guarantor of the study and accepts full responsiblity for the work and the conduct of the study, had access to the data, and controlled the decision to publish. JB was a partner investigator, contributed to project planning, design and data analysis, writing and revising of the manuscript, where JP and PK led recruitment at the hospital sites, as well as assisted in manuscript edits. KK conducted a large proportion of interviews and was responsible for triangulating data analyses, data management, manuscript development and editing, and participants feedback. The corresponding author attests that all listed authors meet authorship criteria, and that there are no other individuals whom meet the criteria.

Funding This work was supported by an Australian Research Council Linkage Grant LP170100300.

Competing interests None declared.

Patient and public involvement Patients and/or the public were involved in the design, or conduct, or reporting, or dissemination plans of this research. Refer to the Methods section for further details.

Patient consent for publication Consent obtained directly from patient(s)

Ethics approval Ethical approval was granted through the Prince Charles Hospital (Brisbane, Queensland) Committee HREC/18/QPCH/138.

Provenance and peer review Not commissioned; externally peer reviewed.

Data availability statement Data are available upon reasonable request. Data available from corresponding author upon reasonable request.

Open access This is an open access article distributed in accordance with the Creative Commons Attribution Non Commercial (CC BY-NC 4.0) license, which permits others to distribute, remix, adapt, build upon this work non-commercially, and license their derivative works on different terms, provided the original work is properly cited, appropriate credit is given, any changes made indicated, and the use is non-commercial. See: http://creativecommons.org/licenses/by-nc/4.0/.

\section{ORCID iDs}

Jennifer Broom http://orcid.org/0000-0003-3513-3412

Katherine Kenny http://orcid.org/0000-0001-9088-5671 
Jeffrey J. Post http://orcid.org/0000-0003-1475-9272

Pamela Konecny http://orcid.org/0000-0003-0166-2287

\section{REFERENCES}

1 Cassini A, Högberg LD, Plachouras D, et al. Attributable deaths and disability-adjusted life-years caused by infections with antibioticresistant bacteria in the EU and the European economic area in 2015: a population-level modelling analysis. Lancet Infect Dis 2019;19:56-66.

2 Tsuzuki S, Matsunaga N, Yahara K, et al. National trend of bloodstream infection attributable deaths caused by Staphylococcus aureus and Escherichia coli in Japan. $J$ Infect Chemother 2020;26:367-71.

3 AURA 2019. Third Australian report on antimicrobial use and resistance in human health. Sydney; 2019

4 ACSQHC. Antimicrobial prescribing practice in Australian hospitals: results of the 2018 Hospital national antimicrobial prescribing survey. Sydney: National Centre for Antimicrobial Stewardship and Australian Commission on Safety and Quality in Health Care, 2020.

5 Vandael E, Magerman K, Coenen S, et al. Antibiotic consumption in Belgian acute care hospitals: analysis of the surveillance methodology, consumption evolution 2003 to 2016 and future perspectives. Euro Surveill 2019;24.

6 Gürtler N, Erba A, Giehl C, et al. Appropriateness of antimicrobial prescribing in a Swiss tertiary care Hospital: a repeated point prevalence survey. Swiss Med Wkly 2019;149:w20135.

7 Tersigni C, Montagnani C, D'Argenio P, et al. Antibiotic prescriptions in Italian hospitalised children after serial point prevalence surveys (or pointless prevalence surveys): has anything actually changed over the years? Ital J Pediatr 2019;45:127.

8 Lendingham K. Antibiotic resistance: using cultural contexts of health approach to address a global health challenge. UN City, Marmorvej 51, DK-2100 Copenhagen $\varnothing$, Denmark: World Health Organisation, Europe WROf, 2019.

9 Broom J, Broom A, Kirby E. The drivers of antimicrobial use across institutions, stakeholders and economic settings: a paradigm shift is required for effective optimization. J Antimicrob Chemother 2019;74:2803-9.

10 Kirby E, Broom A, Gibson A, et al. Medical authority, managerial power and political will: a Bourdieusian analysis of antibiotics in the hospital. Health 2018;22:500-18.

11 Charani E, Smith I, Skodvin B, et al. Investigating the cultural and contextual determinants of antimicrobial stewardship programmes across low-, middle- and high-income countries-A qualitative study. PLoS One 2019;14:e0209847.

12 Broom A, Kenny K, Prainsack B. Antimicrobial resistance as a problem of values? views from three continents. Crit Public Health 2020;31:1-13.

13 Keizer J, Beerlage-De Jong N, Al Naiemi N, et al. Finding the match between healthcare worker and expert for optimal audit and feedback on antimicrobial resistance prevention measures. Antimicrob Resist Infect Control 2020;9:125.

14 Yoong SL, Hall A, Stacey F, et al. Nudge strategies to improve healthcare providers' implementation of evidence-based guidelines, policies and practices: a systematic review of trials included within Cochrane systematic reviews. Implement Sci 2020;15:50.
15 Roope LSJ, Buchanan J, Morrell L, et al. Why do Hospital prescribers continue antibiotics when it is safe to stop? results of a choice experiment survey. BMC Med 2020;18:196.

16 Broom A, Gibson AF, Broom J, et al. Optimizing antibiotic usage in hospitals: a qualitative study of the perspectives of hospital managers. J Hosp Infect 2016;94:230-5.

17 Klein EY, Van Boeckel TP, Martinez EM, et al. Global increase and geographic convergence in antibiotic consumption between 2000 and 2015. Proc Natl Acad Sci U S A 2018;115:E3463-70.

18 Shrestha P, Cooper BS, Coast J, et al. Enumerating the economic cost of antimicrobial resistance per antibiotic consumed to inform the evaluation of interventions affecting their use. Antimicrob Resist Infect Control 2018;7:98.

19 Rogers Van Katwyk S, Grimshaw JM, Nkangu M, et al. Government policy interventions to reduce human antimicrobial use: a systematic review and evidence MAP. PLoS Med 2019;16:e1002819.

20 Broom A, Broom J, Kirby E. Cultures of resistance? A Bourdieusian analysis of doctors' antibiotic prescribing. Soc Sci Med 2014;110:81-8.

21 Broom J, Broom A, Plage S, et al. Barriers to uptake of antimicrobial advice in a UK Hospital: a qualitative study. J Hosp Infect 2016;93:418-22.

22 Gale NK, Heath G, Cameron E, et al. Using the framework method for the analysis of qualitative data in multi-disciplinary health research. BMC Med Res Methodol 2013;13:117.

23 National antimicrobial resistance strategy. Commonwealth of Australia; 2015.

24 UK five year antimicrobial resistance strategy 2013-2018. London: Department of Health 2013.

25 McLeod M, Ahmad R, Shebl NA, et al. A whole-health-economy approach to antimicrobial stewardship: analysis of current models and future direction. PLoS Med 2019;16:e1002774.

26 Aarons GA, Hurlburt M, Horwitz SM. Advancing a conceptual model of evidence-based practice implementation in public service sectors. Adm Policy Ment Health 2011;38:4-23.

27 Braithwaite J, Churruca K, Long JC, et al. When complexity science meets implementation science: a theoretical and empirical analysis of systems change. BMC Med 2018;16:63.

28 Wathne JS, Skodvin B, Charani E, et al. Identifying targets for antibiotic stewardship interventions through analysis of the antibiotic prescribing process in hospitals - a multicentre observational cohort study. Antimicrob Resist Infect Control 2020;9:114.

29 Evans CD, Lewis JWS. Collaborative antimicrobial stewardship in the health department. Infect Dis Clin North Am 2020;34:145-60.

30 Van Katwyk SR, Grimshaw JM, Hoffman SJ. Ten years of inaction on antimicrobial resistance: an environmental scan of policies in Canada from 2008 to 2018. Healthc Policy 2020;15:48-62.

31 European Centre for Disease Prevention and Control. Ecdc country visit to Italy to discuss antimicrobial resistance issues. 2017, 2017. Available: https://ecdc.europa.eu/en/publications-data/ecdccountry-visit-italy-discuss-antimicrobialresistance-issues [Accessed 13 Aug 2020].

32 Courtenay M, Castro-Sanchez E, Fitzpatrick M, et al. Tackling antimicrobial resistance 2019-2024 - The UK's five-year national action plan. J Hosp Infect 2019;101:426-7.

33 Australia's National Antimicrobial Resistance Strategy 2020 \& Beyond. Canberra: Department of Health, Commonwealth of Australia 2019. 